Discours homophobe.

\title{
Le témoignage comme discours alternatif
}

Claudine Moïse et Claire Hugonnier

Univ. Grenoble Alpes, LIDILEM, 38000 Grenoble, France

Proposition revue SEMEN, ${ }^{\circ} 47$.

\section{Discours de la haine dissimulée. Quelles stratégies de contre-discours}

\section{Axe 2. Stratégies construisant les contre-discours}

\section{Résumé}

En nous appuyant sur un terrain ethnographique mené au sein d'une association à Grenoble, nous analyserons les propositions de contre-discours émanant de la structure dont l'objectif est de faire face aux discours homophobes circulants. Notre corpus est constitué de 13 témoignages écrits de jeunes victimes d'homophobie, diffusés sur le site internet de l'association. Le témoignage de l'expérience traumatique est un genre discursif largement employé pour permettre la transmission d'une mémoire, faciliter la résilience pour les victimes mais aussi pour prévenir les discours de haine.

À partir des différentes réflexions autour du discours de haine homophobe, notamment implicite, détourné ou dissimulé, et après avoir théorisé, d'un point de vue des hégémonies et des idéologies, la notion complexe de contre-discours, nous adopterons la notion de discours alternatif plutôt que celle de contre-discours. Il s'agira pour nous de montrer que le témoignage peut être une forme de discours alternatif à l'homophobie. Sans être dans l'analyse de la réception, nous verrons alors comment ce discours est construit, quelles sont ses caractéristiques argumentatives, et plus précisément la forme de mise en récit de de processus pathémiques qu'il déploie. Nous essaierons de montrer pourquoi, par ces effets argumentatifs, le témoignage peut être un genre de discours alternatif possible au discours de haine.

\section{Mots clé}

ethnographie, discours alternatif, homophobie, sociolinguistique, témoignage

\begin{abstract}
Based on an ethnographic field achieved within an association in Grenoble, we will analyze the counterdiscourse emanating from the structure whose goal is to cope with circulating homophobic discourse. Our corpus is made up of 13 written testimonials of young victims of homophobia diffused on the website of the association. The testimony of the traumatic experience is a discursive genre widely used to allow the transmission of memory, to facilitate resilience for the victims but also to prevent hate speech.

From the various reflections around homophobic hate speech, implicitly, diverted or concealed, and after having theorized, from a point of view of hegemonies and ideologies, the complex notion of counter-speech, we will adopt the notion of alternative speech rather than that of counter-speech. The aim is to demonstrate that testimony can be a form of alternative speech to homophobia. Without being in the analysis of the reception, we will then see how this discourse is constructed, while investigating its argumentative characteristics, and more precisely the form of narrative of pathemic processes that this discursive genre deploys. We will try to explain why, through these argumentative effects, testimony may be a kind of possible alternative discourse to hate speech.
\end{abstract}




\section{Key words}

alternative speech, ethnography, homophobia, sociolinguistics, testimony

\section{Introduction}

Le discours de haine a été défini par le Comité des Ministres du Conseil de l'Europe comme « couvrant toutes formes d'expression qui propagent, incitent à, promeuvent ou justifient la haine raciale, la xénophobie, l'antisémitisme ou d'autres formes de haine fondées sur l'intolérance, y compris l'intolérance qui s'exprime sous forme de nationalisme agressif et d'ethnocentrisme, de discrimination et d'hostilité à l'encontre des minorités, des immigrés et des personnes issues de l'immigration » (Weber, $2008: 3$ ). En ce sens, et malgré une définition qui peut être jugée approximative et peu précise, le discours de haine concerne la haine ethnique, raciale, religieuse, de genre - à l'égard des femmes en particulier, les propos homophobes, la menace pour l'ordre démocratique, le négationnisme et le révisionnisme, l'apologie des crimes de guerre, de la violence et du terrorisme ${ }^{1}$. Au vu de la multiplicité des catégories concernées, nous nous intéresserons plus précisément, au discours homophobe, à ses caractéristiques énonciatives, notamment telles qu'elles sont reprises dans les témoignages qui relatent des expériences d'homophobie.

Dans le cadre du groupe de recherche Draine ${ }^{2}$ Haine et rupture sociale, discours et performativité, qui cherche à définir le discours de haine et à analyser ses contre-discours, nous avons caractérisé le discours de haine directe par les phénomènes discursifs conjoints d'exclusion-négation-anéantissement, d'actes de condamnation et d'emphases pathémiques qui sont à relier aux procédés utilisés dans la violence verbale fulgurante. Nous avons vu aussi que la haine pouvait s'inscrire dans des formes discursives indirectes, comme par exemple la dénégation. Nous verrons ici à travers nos données comment s'expriment les marques homophobes directes mais aussi indirectes.

Ainsi, en regard des discours homophobes, nous nous intéresserons ici plus particulièrement au témoignage, qui peut servir leur prévention. Quelles sont les stratégies discursives du témoignage pour répondre à l'homophobie et mieux la contrer? En quoi ces discours

\footnotetext{
${ }^{1}$ Pour notre propos nous adopterons la définition suivante même si légalement les définitions n'incluent pas toujours ces catégories (cf. l'introduction de ce volume).

2 Projet H2020, PRACTICIES, Partnership Against The Radicalization onlIne of Youths Subjects pour lequel Claudine Moïse est responsable d'un workpackage et autour duquel elle a constitué un groupe de recherche international, Draine, en analyse de discours, sur les discours de haine.
} 
s'apparentent à des discours que l'on peut appeler alternatifs, à un discours autre ? Pourquoi ne sont-ils pas des contre-discours?

Pour ce faire, nous interrogerons tout d'abord le genre du témoignage face aux notions de contre-discours et de discours alternatif. Nous verrons ensuite les caractéristiques discursives du témoignage, effet véridictoire objectivé dans une mise en tension, qui en font un discours alternatif bien éloigné d'un discours de haine.

En nous appuyant sur un terrain ethnographique mené au sein de l'association Le Refuge ${ }^{3}$ à Grenoble, nous analyserons 13 témoignages écrits de jeunes, diffusés sur le site de l'association pour contrer les comportements et les discours homophobes.

\section{Une étude ethnographique}

\subsection{Le cadre de l'étude}

En 2003, Nicolas Noguier, sensible à la situation d'exclusion de jeunes homesexuelles et homosexuels et conscient du manque de lieux d'accueil en France, décide de créer Le Refuge. La structure, dont il est désormais président, s'implante dans un premier temps à Montpellier et compte aujourd'hui dix-huit délégations en France métropolitaine et dans les Dom-Tom. Elle a pour mission de venir en aide aux jeunes de 18 à 25 ans qui se retrouvent en situation d'errance à la suite d'une rupture familiale causée par la révélation de leur orientation sexuelle. L'annonce de l'homosexualité ou de la transidentité de leur enfant est parfois vécue par les parents comme un choc et les jeunes se retrouvent sujets à des violences physiques et psychologiques, causées par l'ensemble des membres de leur famille. En effet, si pour certaines et certains jeunes la mise à la porte du domicile familial est rapide et directe, d'autres subissent jusqu'à leur majorité de lourdes pressions sociales. Le Refuge leur propose alors un hébergement temporaire et un accompagnement social, psychologique, médical et juridique afin d'entrevoir l'avenir de manière plus paisible. Les statistiques du Rapport d'Activité 2017 du Refuge montrent que les profils les plus représentés au sein des hébergé·e·s sont des adolescentes et adolescents de milieux modestes, de familles religieuses pratiquantes et d'un parcours d'aide social à l'enfance. Dans ce même document, Le Refuge dit accueillir des personnes en souffrance, isolées et en manque d'estime d'elles-mêmes. En effet, ce rapport

\footnotetext{
${ }^{3} \mathrm{https}: / /$ www.le-refuge.org/l-association/
} 
montre qu'avant de franchir la porte, la plupart des jeunes a connu la rue, la consommation abusive de drogues et d'alcool, a pratiqué des comportements sexuels à risques ou a tenté de se suicider. Si Le Refuge a pour objectif d'assurer un service d'accueil, d'écoute, de soutien et de médiation familiale, l'association s'est également engagée dans la lutte contre l'homophobie par le biais de sensibilisation des acteurs publics ou privés.

\subsection{L'enquête ethnographique et les données}

L'association Le Refuge Isère est apparue comme un terrain idéal de recueil de données sur le discours de haine et sur ses contre-discours ${ }^{4}$. À partir d'un travail ethnographique mené pendant six mois (de janvier à juin 2018), il s'est agi d'analyser de quelles façons l'association tentait de lutter contre l'homophobie, et, plus précisément, de voir quelles étaient les différentes propositions de discours de prévention émanant de l'association - sensibilisation en milieu scolaire, affiches, livrets, témoignages - pour faire face aux discours homophobes circulants. À partir de ce travail, un large corpus de données textuelles a été constitué. Pour cet article, nous avons choisi d'étudier treize témoignages de jeunes hébergé·e·s et suivi·e·s par Le Refuge, textes provenant du site de l'association (https://www.le-refuge.org/actions/testimonial.html). Selon le chargé de communication, les témoignages sont le résultat d'une écriture spontanée, toutefois corrigée par lui-même pour ce qui est de l'expression. Les textes ont été aussi harmonisés pour présenter une certaine cohérence. Parmi ces prises de parole, nous avons écarté les témoignages qui faisaient état d'un parcours de transition, et ceux qui étaient davantage des demandes de conseils. En raison de l'hétérogénéité des contenus des récits, nous n'avons pas ici étudié les témoignages chacun dans leur spécificité propre mais nous avons tenté de dégager un fonctionnement discursif récurrent pouvant s'apparenter à ce qui serait un discours alternatif.

\section{Le témoignage comme discours alternatif}

\section{1. De la polémique au contre-discours}

L'argumentation polémique (Amossy, 2014) en jeu dans les interactions est un type particulier de discours ou d'échanges, basé sur la controverse voire le conflit (Moïse, Romain, 2010 ;

\footnotetext{
${ }^{4}$ Ce travail a fait l'objet d'un mémoire de Master 2 en Sciences du Langage, intitulé Milieu associatif et conflits : tensions, médiations, résolutions. Le Refuge à Grenoble, dans le cadre du parcours « Médiations langagière et culturelle en situation de conflit ».
} 
Moïse, 2016) ; elle s'appuie sur des figures rhétoriques caractérisées (l'exemple, l'ironie, la définition polémique qui donne une définition orientée d'emblée), la réfutation qui mobilise non seulement des contre-arguments mais des arguments ad hominem, l'assertion, formes de procédés évaluatifs et métadiscursifs de son propre discours, voire des formes de l'agression (sarcasme, injure), etc. (Moïse, Romain, 2010). Les procédés argumentatifs polémiques alimentent la controverse dans une visée persuasive mais reposent aussi sur des effets de pathos, élément traditionnel de la rhétorique (Rinn, 2008). Le pathos est l'effet émotionnel produit sur l'allocutaire (ce n'est pas systématiquement celui ressenti par le sujet parlant) et le recours aux émotions, dans un cadre de connivence, sert autant le polémiste à convaincre son auditoire de ses bons arguments qu'à susciter à son égard une certaine compassion.

La polémique serait alors au centre du contre-discours (Plantin, 1996) dans le sens où la confrontation argumentative, autour de la divergence de points de vue sur une même question, serait une volonté de convaincre son interlocuteur voire un tiers, à tout prix et par tous les moyens. Le contre-discours se construit dans une opposition argumentative vive et émotionnelle, entre réfutation, confrontation et remise en cause, pouvant réactiver les arguments voire les attaques du discours source.

Ainsi, les procédés discursifs polémiques des contre-discours ne peuvent, de notre point de vue, se départir des enjeux interpersonnels des locuteurs et être dissociés des besoins de reconnaissance et d'identité. Ils s'inscrivent dans une absence de négociation ou dans le maintien d'une forte dissymétrie ; les ressources et processus langagiers déployés, qui relèvent de processus identitaires et de stratégies discursives de résistance et de contre-pouvoir interactionnels, permettent alors aux acteurs d'une interaction de se positionner vis-à-vis de l'autre. Dans une telle perspective, l'argumentation/contre-argumentation se co-construit entre les interlocuteurs et s'actualise dans un va-et-vient nécessaire (arguments contre arguments, attitudes contre attitudes, rapports de face contre rapports de face). En ce sens, les interactions polémiques en contre-discours jouent sur la confrontation, dans une perspective d'échanges argumentatifs pour, en général, obtenir adhésion et soutien de ceux qui écoutent, et où se jouent gestion des faces (entre attaque et préservation) et guerre de positions.

\subsection{Le genre du témoignage}

En regard de cette définition du contre-discours, le témoignage ne semble pas emprunter les mêmes stratégies et visées discursives ; il apparaît même comme tout autre. Objet d'études, notamment en Histoire (Torterat, 2017) ou en littérature autour de l'esthétique (Dulong, Dormier (éds), 2005 ; Rastier, 2010 ; Detue, Lacoste (éds), 2016), alors qu'il porte souvent sur 
des événements traumatiques et tragiques comme la Shoah, la guerre d'Algérie, ou aujourd'hui les attentats islamistes, il repose sur des visées de transmission et d'expression de soi. Qu'ils soient diffusés à partir de reportages, de films, sur les sites internet, d'essais littéraires, entre parler pour les autres et parler pour soi, les témoignages tentent de changer les représentations premières et émotives (Vous n'aurez pas ma haine, témoigne Antoine Leiris, 2016), de proposer un autre point de vue sur les événements, de les expliquer autrement, de donner à réfléchir. Il ne s'agit pas de contrer frontalement les arguments des discours premiers, par exemple pour nous ici les discours homophobes cités, mais d'apporter, par des expériences de vie, une autre vision du monde.

En ce sens, il s'agit de faire œuvre de transmission pour rester en alerte quand se joue la transformation de l'individuel vers le collectif dans un acte qui se fait politique. « Le but communicatif ne consiste pas seulement à témoigner d'une expérience véridique, mais à promouvoir un projet de société, susceptible de valider de nouvelles règles éthiques » (Rinn, 2015 : 2). De cette façon, dans un travail de mémoire (Mayaffre, Ben Hamed, 2014), le témoignage rend aussi hommage contre l'oubli à l'ensemble des victimes ayant vécu les mêmes événements douloureux. Il permet aussi de se dire dans une libération de la parole face au trauma, sans guerre de positionnement identitaire.

Pour remplir toutes ces fonctions de conscientisation et de transmission sociales, le témoignage doit s'appuyer sur une visée véridictoire où les émotions serviront son propos, sans toutefois débordement émotionnel polémique. Le témoignage, selon « la tradition judiciaire » (Rastier, 2010 : 115) devra s'accrocher aux faits, à la vie objectivée, au-delà de toute sentimentalité pathémique, sans toutefois oublier de toucher l'interlocutrice ou interlocuteur :

« le témoignage adopte en général une esthétique "classique » et un réalisme empirique : il procède par choix scrupuleux, avec une volonté de mettre en relief l'essentiel, d'éviter le «tout dire », le déballage, la surenchère. D'humbles détails, qu'aucun faussaire ne pourrait imaginer, révèlent l'impensable : et cependant, la maîtrise surmonte la hantise. C'est d'ailleurs pour cela que certains témoignages sont devenus des classiques : adressés à l'humanité, ils prennent une valeur universelle » (Raster, $2010: 117$ )

Ainsi, à partir de ces éléments, il est possible d'affirmer que les caractéristiques fondamentales du contre-discours ne se retrouvent pas au sein du genre discursif du témoignage. En effet, le témoignage ne répond pas à une volonté d'opposition, de polémique et de controverse ; et c'est bien de cette façon, que ce discours, loin d'un contre-discours se fera discours alternatif. 


\section{Les témoignages de l'association Le Refuge, discours alternatifs}

Selon les responsables de l'association, les témoignages sur le site du Refuge ont pour objectif de permettre à certains jeunes de se sentir proches de parcours de vie et de faciliter ainsi la prise de contact avec les membres du groupe, mais aussi de rendre compte d'expériences pour contrer l'homophobie. De cette façon, et pour convaincre, le témoignage, à l'image du genre qu'il incarne et comme discours alternatif, doit exprimer une vérité concrète et des émotions objectivées, toucher son auditoire, se distancier du discours de haine homophobe.

\section{1. Un récit véridictoire}

Le témoignage, sous forme de narration, va user des caractéristiques du récit oral (Adam, 1994 ; Brès, 1994) dans une intention de vérité et à partir de deux procédés essentiels, une construction narrative autour de faits précis et crédibles et des éléments émotionnels objectivés.

\section{La construction narrative}

S'ils ont été harmonisés et s'ils sont tous présentés de la même manière par le personnel du Refuge, en six temps, présentation de soi, contexte familial, prise de conscience de son homosexualité, révélation de l'homosexualité, conséquences dramatiques, rencontre avec $L e$ Refuge, ces récits se caractérisent par des récurrences narratives et une logique causative, qui montre, avec force persuasion, l'enchaînement des événements. Leur répétition, donc leur généralisation, ancre ces témoignages dans une expérience et une vérité partagées et à faire partager.

La moitié des jeunes qui se racontent commencent par se présenter de façon factuelle, ce qui permet de bien identifier l'acteur-sujet du récit. Au-delà du prénom et de l'âge, certain·e·s développent un peu plus autour de leur ville d'origine ou de leur enfance.

\section{Exemple 1. Julien : Je suis né le 2 juillet 1994 en Seine-et-Marne, dans une petite ville qui s'appelle Salins. J'y ai habité jusqu'à l'âge de mes 10 ans}

Suit le contexte familial et/ou amical évoqué qui permet de situer le récit, d'en donner le cadre et le contexte.

Exemple 2. Alain : Abandonné à la naissance, j'ai été placé en famille d'accueil à l'âge d'un an. Je voyais ma famille biologique de temps en temps, lors de visites encadrées. J'ai pu aller chez ma mère un week-end par mois, mais ça se passait très mal et elle nous battait, mes sæurs et moi : à mes 11 ans, les visites ont été arrêtées sur ordonnance du juge. Ma famille d'accueil est devenue « Ma Famille ».

Puis, la prise de conscience représente un événement qui bouleverse le cours de l'enfance ou de l'adolescence et qui va faire intrigue dans le fil du récit. Cet épisode de vie est intéressant 
dans sa complexité et apporte, malgré sa répétition, des singularités aux prises de parole. Les récits permettent de redonner une épaisseur humaine aux différentes expériences de vie, pour asseoir une vérité dans ses subjectivités énonciatives. Entre le fait de penser à une maladie (Julien), de se demander si on est normal, si on est seul dans ce cas-là (Matthieu), d'être confronté à une famille homophobe (Gildas, Jordan), d'être tentée par le suicide (Denise), de faire face aux manifestations contre le mariage pour tous (Loïc), de se poser beaucoup de questions (Jordan, Alain, Alex, Jessy), ces moments sont vécus la plupart du temps dans la souffrance ou dans la dissimulation, comme l'expriment Matthieu, Jordan ou Romain. De la singularité à la mêmeté, les témoignages s'inscrivent dans des récurrences empiriques, des faits de vie assez répandus qu'on ne peut ignorer.

\begin{abstract}
Exemple 3. Matthieu : ’̀ 14 ans, j'étais en 4 e, je me sentais très proche des filles, mais je n'avais aucune attirance envers elles. Je me posais énormément de questions. On ne m'a jamais parlé de l'homosexualité, je me demandais si j'étais le seul à ressentir de telles choses, je me demandais si j'étais normal, je me demandais pourquoi moi ? Pensant que ça allait passer, je n'ai rien dit à personne, je jouais à «l'hétéro ».
\end{abstract}

La prise de conscience va ouvrir sur la révélation, personnelle ou imposée par d'autres. Là encore, les témoignages, à partir d'un même événement, présentent une diversité de situations et de tensions singulières selon que l'on agit ou que l'on est agi. Ces différences événementielles confortent l'individuation dans des régularités narratives, ce qui pose des vérités personnelles dans une plus vaste qui les englobe. Les coming-out permettent au sujet de se sentir responsable et leur expression sont souvent brèves et affirmées (Julien, Matthieu, Denise, Romain, Jordan, Angello, Jessy) comme l'exprime Jordan. En revanche, la découverte par un tiers de l'homosexualité, acte fort de condamnation, demande au sujet de développer son récit pour exprimer précisément le contexte de la découverte (Gildas, Alex, Alain, Michel, Thomas).

\footnotetext{
Exemple 4. Gildas. Un jour où mon copain me ramenait au lycée, alors que nous étions main dans la main dans une rue où il n'y avait presque personne, j'ai croisé mon oncle. [...] Le lendemain, en allant sur Facebook, j'ai découvert un message de ma mère, à qui je ne parlais plus depuis plus de 3 ans : "I'ai eu Christopher hier. Il m'a dit qu'il t'avait croisé avec un mec, apparemment vous vous teniez la main. Si tu avais été éduqué par moi, tu ne serais jamais devenu "PD". Tu es la honte de la famille. Tu peux dire que je n'ai pas été une bonne mère, mais alors toi, tu es un "PD". [...] ».
}

Les conséquences de la révélation de l'homosexualité sont dramatiques et les récits décrivent assez longuement des faits tragiques entre rejet, discours homophobes, passage à la rue, tentatives de suicide. Parmi les actions mentionnées on trouve l'enfermement dans sa chambre, le fait de manger à part, le placement en foyer d'accueil, la prostitution, la drogue, les tentatives 
de suicide, la violence physique, faits douloureux par leur concrétude et leur renvoi au réel. Un extrait du récit de Jordan en donne un exemple :

\begin{abstract}
Exemple 5. Jordan : Le lendemain, tout le collège savait que j'étais gay, et c'est là que tout a changé. D'insultes homophobes aux violences physiques, j'ai passé la plus dure journée de toute ma vie. Le lendemain, rebelote, et c'est là que j'ai craqué et que tout a basculé : j'ai tenté de me suicider parce que je ne pouvais pas vivre ainsi, avec tant de souffrances en moi. Tout un collège de quartier qui me torture, en plus de ma famille homophobe, c'était difficile...
\end{abstract}

Finalement, l'apaisement et la renaissance viendront avec la rencontre avec Le Refuge. Contact trouvé sur internet, mais le plus souvent donné par une amie ou un ami, Le Refuge sera la dernière étape, celle du réconfort et de l'amour. L'équipe du Refuge apporte écoute, bienveillance, compréhension, et amour pour devenir une nouvelle famille. C'est un temps aussi de reconstruction scolaire, professionnelle et amoureuse.

\title{
L'objectivation des émotions
}

Les faits chronologiques, construits dans un objectif de logique véridictoire, sont évoqués sans effets d'hyperbole ou d'emphase. À travers les événements racontés, les détails du réel, en guise de contrat testimonial matérialiste, composent une " exigence de concrétude - et d'exactitude - [...] garant[s] de la valeur de vérité du texte, $[\ldots]$, « voué[s] à déjouer une abstraction » (Detue, Lacoste, $2016: 8$ ). La structuration du récit s'appuie sur une objectivation praxéologique et une distanciation à soi, les moments douloureux sont suggérés de façon directe sans ressenti intime, pour en accentuer la violence vécue. S'ils relèvent d'une axiologie négative, les mots utilisés nomment les faits du réel avec « réalisme empirique ». Par exemple, on peut relever dans les passages sur les contextes familiaux, des termes très précis et évocateurs, tels violence (3 fois), alcoolique (2 fois), boire, menace, soumise, quitter, partir, strict, manipulation, cris, pleurs, colère, misérable, battre (2 fois), enfer, bonniche, souffert (2 fois), difficile (2 fois), disputer, rejet, vide, abandon qui font référence à des actes et non à des jugements ou des sentiments plus abstraits. De même les conséquences de la révélation de l'homosexualité sont mentionnées autour d'agissements tragiques, concrets, dans un principe de réalité, entre rejet, discours homophobes, passage à la rue, tentatives de suicide. La force des témoignages est de ne jamais accuser, dénoncer, attaquer mais d'exposer les faits bruts, ce qui permet d'échapper à une pathémisation qui basculerait dans l'épanchement fusionnel.

L'objectivation émotionnelle passe par le filtre de la mémoire dans le sens où la souffrance est revue à l'aune d'un discours plus rationnel, spécifique et spécialisé autour des termes comme homophobie, maltraitance, main courante, être coupable, victime, être faible moralement, fils 
biologique, ceux sans doute acquis par la suite autour des questions d'homosexualité et d'homophobie. Ce va-et-vient constitue une « sédimentation mémorielle dans laquelle s'accumulent et se combinent souvenirs intimes du témoin et narration historique ou collective » (Mayaffre et Ben Hamed, 2014 : 2). Cet effet discursif accentue encore la distanciation à soi et le principe de réalité concrète.

Exemple 6. Julien : Alors que ce n'était pas rose, ça n'a fait qu'empirer. Mon beau-père étant homophobe ${ }^{5}$, j'ai encore plus subi sa maltraitance.

Exemple 7. Matthieu : Ils m'expliquent qu'un mois après avoir quitté le domicile de mes parents, ma mère a fait une main courante pour signaler que j'étais parti. Étant mineur, je n'ai pas le choix, je dois suivre les policiers. Je leur raconte tout, je me sens mal, j'ai l'impression d'être coupable de quelque chose alors que je ne suis qu'une victime.

\section{2. La mise en tension narrative}

Cette recherche des faits concrets, de réalisme et d'objectivation ne va pas pourtant sans une volonté d'effets émotionnels chez l'interlocuteur dans la mesure où il s'agit de sensibiliser aux effets de l'homophobie. Cette construction, au-delà, d'une logique causative, qui montre l'enchaînement des événements, manifeste une tension émotionnelle (Adam, 1994 : 431-433) qui prend forme entre une intrigue et une adresse narratives pour l'autre qui lit ou écoute le témoignage.

\section{L'intrigue narrative}

Au-delà du caractère de vérité, le témoignage doit retenir l'attention par l'émotion et donc se construire sous forme d'intrigue. À partir de la prise de conscience de sa propre homosexualité, comment va se passer le questionnement et bouleversement intérieurs autour de la sexualité ? Quelle va être sa résolution? Que va-t-il se passer ? Comment va se résoudre la souffrance ?

Cette intrigue se joue entre «l'unité du récit » (Adam, 1994), un dispositif d'enchainements de cause à effet et «un procès transformationnel». L'unité du récit est constituée par un protagoniste bien identifié, ici le jeune qui se raconte, et, comme nous l'avons vu une succession d'événements en cohérence les uns par rapport aux autres. Les enchaînements sont largement expliqués et marqués par des connecteurs ou des marqueurs temporels comme $c$ 'est là que ou c'est à ce moment que. Ces moments de changements forment un «procès transformationnel », c'est-à-dire que l'action principale (le rejet des jeunes gens), autour d'une histoire (un début - un milieu - une fin), va se transformer pour trouver un certain apaisement

\footnotetext{
${ }^{5}$ Les soulignements sont de nous
} 
(la rencontre avec Le Refuge). La "succession thématique », suite chronologique des événements qui sont reliés et orientés vers une fin, va permettre de relâcher la tension ressentie tout au long du récit. Nous avons ici dans une forme de dramaturgie qui va de la prise de conscience de l'homosexualité, sa révélation et ses conséquences à, en fort contraste dans un retournement en miroir, un dénouement heureux grâce au Refuge. Dans les textes que nous avons analysés, les mots pour qualifier Le Refuge sont dans une axiologie positive (accueillir (4 fois), réconfort (2 fois), soutien (7 fois), rassurer (5 fois), aimer (5 fois), écouter ( 8 fois), être compris ( 5 fois), conseiller ( 5 fois), cour (5 fois), nouvelle famille (16 fois) et le récit se laisse aller, à ce moment-là, à une certaine effusion, marquée notamment par des points d'exclamation et des intensificateurs, tels très, aucun, toutes et tous. En ce sens le récit de Romain est représentatif de ces effets discursifs avec des termes comme « belle équipe » « c'est une famille pour moi ! » et un usage répété des points d'éxclamation :

Exemple 8. Romain : C'est à ce moment qu'une amie très proche m'a parlé du Refuge. Très craintif, j'ai quand même envoyé un SMS sur la ligne d'urgence. J'ai reçu très rapidement une réponse et une semaine plus tard, j'intégrais la délégation de Lille où la belle équipe du Refuge m'aide jour après jour. Bien plus qu'une équipe, c'est une famille pour moi! J'y suis depuis quatre mois, j'ai repris mes études à la fac et j'ai un emploi à mi-temps à la mairie de Lille. Jour après jour, j'essaye de reconstruire ma vie, que je partage aujourd'hui avec quelqu'un. J'ai vingt ans, je n'ai plus aucun contact avec ma famille et ce n'est pas plus mal. Un grand merci à toutes les personnes mobilisées pour nous venir en aide!

\section{Le récit adressé}

La mise en tension de la narration testimoniale est aussi prise de parole à l'égard d'un autre (Labov, 1976, 1981), dans une mission de sensibilisation, conscientisation, transmission. Le témoignage se veut donc adressé à un autre qui écoute ou qui lit. On peut trouver dans les récits, notamment oraux, ce que Labov appelle « le résumé », qui vient parfois en amont du récit à proprement parler, qui n'est pas un condensé de l'histoire, ni une façon de l'annoncer mais d'en montrer l'intérêt. Le résumé « sert à légitimer par avance, en suscitant l'intérêt de cette prise de parole qui est une prise de la parole » (Brès, 1994 : 79). Les jeunes qui témoignent sont rares à l'utiliser comme si le seul fait de témoigner sur le site du Refuge suffisait à apporter crédit et légitimité au témoignage.

En revanche, l'adresse prend d'autres formes et vise deux cibles, celle ou celui qui va lire le témoignage et les membres du Refuge. L'adresse à l'interlocutrice ou à l'interlocuteur, même si elle est rare, mérite d'être signalée dans la mesure où elle crée un mouvement de complicité et de connivence, particulièrement quand elle prend la forme d'un aveu, je ne vous cache pas dit Alex, ou d'une antiphrase, je ne sais pas si vous pouvez imaginer dit Adrien. L'adresse est 
aussi celle envers Le Refuge, qui dans une forme de coda ${ }^{6}$, dit un certain épanchement en remerciements et gratitude, en rupture de ton avec le reste du témoignage, ce qui permet de résoudre la tension en terminant par un apaisement de fin de récit. Le texte de Matthieu en serait un prototype, avec ses répétitions, grâce à vous, son axiologie positive, cœur, aimer, avancer, bâtir, vie, ses emphases, plus jamais, enfin.

Exemple 8. Matthieu : Vous avez tous une place dans mon cour. Il faut encore que j'apprenne à aimer mon corps, à m'aimer, tout simplement, grâce à vous, je sais que je ne serais plus jamais seul, grâce à vous, je peux enfin avancer et bâtir ma vie.

Ainsi, les témoignages par leur ancrage dans les détails du réel, leur construction narrative et l'objectivation des émotions ont valeur de vérité ; par une mise en intrigue adressée à l'interlocutrice ou l'interlocuteur, ils permettent aussi un partage des émotions sans débordement affectif. Ils donnent à voir un retournement d'homophobie vécue sans entrer toutefois dans une opposition polémique, sans se faire contre-discours haineux mais discours de sensibilisation alternatif.

\section{3. Le témoignage face et contre le discours de haine homophobe}

Les témoignages des jeunes ne contrent pas directement les discours homophobes. Discours de vérité objectivée, leurs témoignages vont également dénoncer l'homophobie en la racontant, mais sans reproduire en retour un discours haineux. Depuis les théories bakhtiennes du dialogisme, le discours est toujours porté par différentes voix saisissables dans un même énoncé. Le discours rapporté (Rosier, 2004), largement utilisé dans les témoignages qui nous occupent, élément de dialogisme, permet de reprendre certains discours homophobes tout en les renversant.

\section{Les marques homophobes directes}

Dans l'intégralité des témoignages, les jeunes mettent en scène l'homophobie dont elles et ils ont été victimes. Par l'emploi du discours rapporté, elles et ils font appel aux propos homophobes de leur entourage amical, familial et professionnel et cette stratégie discursive alimente la construction d'un récit de vérité. Comme nous allons le développer, ils mettent en circulation des discours aux marques homophobes directes, l'expression de l'exclusion, les actes de condamnation et l'expression de la honte, éléments constitutifs d'un discours de haine (Lorenzi et Moïse (éds) à paraître).

\footnotetext{
${ }^{6} \mathrm{La} \ll$ coda » marque, par une formule de conclusion, la fin du récit.
} 
L'exclusion

$\mathrm{Au}$ sein des témoignages, le phénomène d'exclusion apparait de manière récurrente et est évoqué à travers le discours rapporté et des injonctions, acte de violence verbale et de rejet de l'autre (Lorenzi, 2015), tels je te vire ou dégage :

Exemple 9. Matthieu : À mon retour, mon père me dit : « soit tu pars, soit je te vire » [...] Mon frère s'avance vers moi et me dit : " tu n'as pas compris, on t'a dit de dégager, donc dégage.

Exemple 10. Alain : Ma mère d'adoption m'a regardé et m'a dit : " dégage ".

Exemple 11. Romain : Jusqu'à ce jour où mon père a sorti un fusil de chasse et l'a braqué vers moi en me disant « maintenant dégage, je n'aime pas les $P D$, les pédales n'ont pas leur place dans cette maison...

Exemple 12. Angelo : Ma mère m'a demandé de partir de la maison, en me disant : «Tu es gay, tu dégages, je ne veux plus de toi».

Cette stratégie discursive a pour objectif de produire un récit de véracité et rendre audible une réalité de souffrance quand, selon le Rapport annuel 2018 de SOS Homophobie, à 62\% l'homophobie s'exprime à travers des situations de rejet et d'ignorance à l'égard de personnes homosexuelles ou supposées l'être.

Si les discours rapportés expriment l'exclusion, le rejet et donc la négation de l'autre, éléments constitutifs du discours de haine homophobe, pour autant les témoignages produits par les jeunes ne sont pas dans des effets de détestation en miroir ou dans des jugements essentialisants. Parce qu'ils ne se posent pas en opposition et ne revendiquent pas non plus une confrontation directe face aux discours sources homophobes, ces témoignages s'éloignent du genre discursif du contre-discours pour se rapprocher davantage d'un discours alternatif, comme nous l'avons défini précédemment, c'est-à-dire qu'il ne s'agit pas de contrer frontalement les arguments des discours premiers, par exemple pour nous ici les discours homophobes cités, mais d'apporter, par des expériences de vie, une autre vision du monde.

Les actes de condamnation

Dans la doxa homophobe, l'homosexuel serait perçu comme un individu dominé, impuissant, efféminé qui menacerait l'ordre «naturel» du monde et ne mériterait ni respect ni considération (Tin, 2003 ; Van Raemdonck, 2011 ; Baider 2017). Ainsi, comme le montrent les discours rapportés dans les témoignages « les mots pour dire l'homosexuel s'avèrent être la plupart du temps les mots de l'homophobie »(Van Raemdonck, 2011 : 185). La mise en 
circulation des désignations telles que tarlouze (2 fois), pédale (2 fois), PD (23 fois) permet d'expliciter la perception négative de homosexualité des jeunes par leur entourage et la façon dont s'exerce précisément l'homophobie dans leur quotidien. L'insulte homophobe vise l'identité sexuée du sujet dans une condamnation violente de tout son être, ce qui renvoie à un des éléments du discours de haine.

Cette forme de condamnation haineuse par l'insulte (Rosier, 2008) va jusqu'à la négationdisparition-anéantissement de l'autre dans une imprécation à mourir. Les témoignages qui donnent à voir indirectement le discours de haine homophobe, relatent les propos de parents qui souhaitent explicitement le décès de leur enfant en raison de leur homosexualité.

Exemple 13. Matthieu : Un jour, mon père me dit : «si j'avais su qu'un jour tu serais $P D, j$ 'aurais demandé à ta mère d'avorter » [...] Il me répond : "de toute façon, un PD ne mérite pas de vivre».

Exemple 14. Gildas: "Tu n'es plus mon fils, je te renie. Ne viens même pas sur ma tombe quand je serais morte, je ne veux pas d'un fils PD. Toute la famille te reniera, tu mérites de crever en enfer $»$.

Exemple 15. Romain : "Vous, les tarlouzes, vous ne méritez pas de vivre »

L'expression de la honte

Émotion puissante et douloureuse, la honte est

« le sentiment d'humiliation provoqué chez une personne, elle rend compte d'un décalage avec des normes sociales attendues vis-à-vis de l'autre, sous le regard de l'autre. Elle exprime chez le sujet visé un sentiment d'infériorité, d'imperfection et provoque un risque d'exclusion, un sentiment de déclassement. Différente de la pudeur, qui consiste à ne pas se montrer, s'exposer, la honte touche l'intégrité du sujet » (Bernard Barbeau et Moïse, 2019).

Certains énoncés rapportés dans les textes des victimes présentent l'homosexualité comme un comportement dont il faudrait avoir honte. Pour aller plus loin, les discours semblent dire que toute personne homosexuelle n'est pas simplement une honte pour elle-même mais également pour les autres. Causer la honte peut provoquer alors chez celle ou celui qui la subit un sentiment puissant de déclassement et, pour s'en défaire, dans un mécanisme de libération, il devient nécessaire de provoquer l'exclusion, la disparition de celui ou celle qui en est la source, soit-il son propre enfant. Ainsi, à travers ces discours, on perçoit de quelles manières l'homosexualité questionne l'image que l'on renvoie aux autres, fondement du sentiment de honte, quand cette image n'entre pas en adéquation avec nos propres valeurs et croyances. Par la convocation des discours antérieurs, les jeunes montrent que le rejet n'a pas simplement été 
matériel mais également symbolique. En effet, à l'annonce de leur homosexualité, les adolescents ont perdu légitimité et estime auprès de leur famille.

Exemple 16. Matthieu : Elle m'a regardé et m'a dit : " tu me fais honte, tu ne peux pas rester comme ça»

Exemple 17. Gildas : lorsque l'on voyait des reportages ou des séries dans lesquelles il y avait des homosexuels, ils disaient : "Regarde-les, les PD, c'est honteux! »

Exemple 18. Alex : elle m'a dit clairement que j'étais la honte de la famille.

\section{Les marques homophobes indirectes}

Il arrive que l'homophobie ne fasse pas l'objet d'une explicitation claire et revendiquée au sein des discours. En effet, certains témoignages de notre corpus font également appel à des propos rapportés qui laissent apparaître des marques homophobes indirectes, de la haine dissimulée, dont les caractéristiques ont bien été explicitées dans Le Dictionnaire de l'homophobie (Tin, 2003 ) et dont nous reprenons ici les grandes lignes.

L'une des premières stratégies des discours homophobes est de s'appuyer sur des éléments étymologiques du mot homosexualité et de se référer avant tout au sexe. Dans les témoignages, à travers les discours rapportés des parents, l'homosexualité est étiquetée à travers le prisme du sexe, au-delà de toute forme d'amour ou de relation. Perçue comme une pratique sexuelle débridée, les parents évoquent certaines pratiques sexuelles brutalement et avec, facteur aggravant, des mots grossiers, ce qui va faire violence : que des "PD", ils n'ont qu'à s'enculer !, T'es allé où? Te faire emmancher? Une seconde stratégie consiste à renvoyer l'homosexualité à ce qu'elle devrait être, une déviance, et à recourir aux normes «naturelles » voire « scientifiques » de la procréation. L'orientation homosexuelle serait selon certains parents haram, honteuse voire pathologique, devant donc être soignée.

Exemple 19. Alex : «la vie, c'est un homme une femme », " t'es malade, faut te faire soigner!»

Exemple 20. Jordan : Une fois dans la voiture, elle m'a remis une claque et s'est mis à m'expliquer que l'homosexualité "c'est mal, que c'est un péché »

Exemple 21. Gildas : "Il faut vraiment que tu ailles en psychiatrie mon fils, tu es un malade mental» 
La dernière stratégie de haine indirecte repose sur un procédé de dénégation ${ }^{7}$, qui consiste à minimiser ou nier les actes et faits historiques homophobes, ce qui permet de minimiser la gravité des agissements des parents, comme le montrent les discours rapportés par Matthieu :

Exemple 22. Matthieu Après appel au procureur de la République et au vu de tous les témoignages, je suis placé six mois dans un foyer. Jour J, ça y est, c'est le jugement, j'ai peur de voir mes parents, peur de la décision du juge. Mes parents passent les premiers et, une fois de plus, minimisent leurs actes, disent qu'ils n'ont rien à se reprocher, qu'ils n'ont rien fait de mal et qu'ils sont de bons parents.

\section{Le discours alternatif au discours homophobe}

La force de ces témoignages, dans une visée empirique de vérité et au-delà d'une expression émotionnelle débordante, est de retenir l'attention dans une forme de compassion. La compassion est ici reliée à la fois à des valeurs morales (on protège ses enfants) et au sentiment d'injustice (Amossy, 2010 : 188). Elle se double d'indignation à l'égard d'un tiers, les parents qui ne sont pas dans leur rôle de protecteur. Mais plus encore, les témoignages remplissent une mission de sensibilisation voire de prévention face au discours homophobe. Non en discours contre mais en discours alternatif. En effet, les témoignages des jeunes ne font pas l'objet de discours polémiques dont la visée serait de produire une controverse face aux discours homophobes. La mise en récit de leur histoire ne constitue pas une parole qui cherche à réfuter les propos d'autrui. Aucune remise en cause de l'adversaire n'est orchestrée. À dire vrai, il semblerait qu'aucun adversaire ne soit présenté dans ces différentes mises en récit. Les personnes aux comportements et paroles jugés homophobes sont avant tout présentées comme un père, une mère, une sœur et/ou un frère. Les témoignages ne font pas état d'une portée virulente, ni d'une volonté d'imposer leur propre vision du réel à leur famille.

Par la narration de leur histoire, les énonciateurs tentent d'introduire une vision autre de l'homosexualité, tout en rendant compte des conséquences de l'homophobie. En convoquant des exemples précis, les témoignages tentent de marquer les esprits. Ainsi, ils montrent avec réalisme la cruauté et la violence qui accompagnent certains propos.

De cette manière, le témoignage des locuteurs ne peut être défini comme une réponse haineuse envers les auteurs de l'homophobie dont ils ont été victimes. En effet, si aujourd'hui il n'y a

\footnotetext{
7 Ces procédés de dénégation peuvent être repris, dans un processus bien connu de minorisation par les minoritaires eux-mêmes, pour tenter de faire disparaitre leur stigmate. En intériorisant le discours homophobe perçu comme légitime, ils peuvent essayer de (se) camoufler leur orientation sexuelle ou être discret, chercher une couverture...
} 
pas de systématisation du discours de haine, les travaux du groupe de recherche Draine ont toutefois permis d'affirmer que ce type de discours se définit par des marques discursives d'altérité fortes dont l'objectif est de déshumaniser l'autre, de le nier jusqu'à l'anéantir. Ce discours offre une vision du monde binaire, appuyée par des processus pathémiques intenses. Or, les différents témoignages de notre corpus ne répondent pas à ces dynamiques. En effet, dans les discours produits par les énonciateurs revendiqués on ne retrouve aucun procédé de catégorisation, d'essentialisation, ni l'utilisation de stéréotypes, d'insultes qui exercerait une mise à distance de l'autre homophobe.

\section{Conclusion. Le témoignage comme transmission sociale et émancipation individuelle}

Grâce à l'analyse de différents témoignages, il a été possible de redéfinir les contours de la notion de contre-discours et de discours alternatif. Alors qu'ils ont la même visée, faire face aux discours de haine, ces types de discours n'empruntent pas les mêmes stratégies, ce qui en fait deux genres discursifs bien distincts. Si le contre-discours se veut dans l'offensive et l'opposition explicitement identifiée et identifiable, le discours alternatif emprunte différents chemins afin d'apporter une autre vision du réel de manière détournée. Finalement, les témoignages des jeunes hébergé.e.s au sein de l'association Le Refuge ont la vertu du genre testimonial. Loin d'être des contre-discours polémiques, ils se veulent discours alternatifs. Ils remplissent une mission mémorielle pour ne pas laisser dans l'oubli la violence vécue, citoyenne et pour tenter de faire évoluer les représentations, cause d'exclusion et de négation de l'autre. Ils ont aussi une valeur intime pour libérer sa propre parole dans une affirmation libératrice de soi.

\section{Références bibliographiques}

ADAM, J.-M., (1994), «Une définition générique du récit ». In BRES, J. (éd), Le récit oral, Montpellier : Praxiling,Université Paul Valéry, Montpellier III , 431-444.

AMOSSY, R., (2010), L'argumentation dans le discours. Paris: Armand Colin.

AMOSSY, R., 2014, Apologie de la polémique. Paris : Presses Universitaires de France.

BAIDER, F., (2017), " "Go to hell you fagots, death to you" Framing the LGBTQ subject in online comments », Lodz Paper in Pragmatics, 14 (1), 69-92.

BERNARD BARBEAU, G. MOÏSE, C., (2019) (à paraître), « Transformation des dynamiques minoritaires, paradigmes sociolinguistiques et émotions ». In Minorisations linguistiques et sociétés / Linguistic Minorities and Society. 
BRÈS, J., (1994), La narrativité. Paris : Editions Duculot.

DETUE, F, LACOSTE, C. (éds), (2016), Témoigner en littérature, Europe, n¹041-1042.

DULONG, R., DORNIER, C. (éds), (2005), Esthétique du témoignage. Paris: Éditions de la Maison des sciences de l'homme.

LABOV, W., (1976), Sociolinguistique. Paris: Minuit.

LABOV, W., (1982), « Speech Actions and Reactions in Personal Narative ». In TANNEN, D. (éd.), Analyzing discourse : text and talk. Georgetown University Round Table, Washington, Georgetown University Press, 219-247.

LEIRIS, A. , (2016), Vous n'aurez pas ma haine. Paris: Fayard.

LORENZI, N., (2015), Violence verbale à l'école. Étude ethnographique et interactionnelle. Thèse de doctorat dirigée par Claudine Moïse, Université Grenoble-Alpes.

MAYAFFRE, D., BEN HAMED, M., (2014), «Récits de mort et souvenir traumatique. Trames et traces lexicales des témoignages sur la Shoah », Argumentation et Analyse du Discours, version en ligne : URL : http://aad.revues.org/1836, page consultée le 2 septembre 2018.

MOÏSE, C., ROMAIN, C., (2010), « Violence verbale et listes de discussions : les argumentations polémiques ». In PIEROZAK I. (éd.), Du « terrain » à la relation : expériences de l'internet et questionnements méthodologiques. Cahiers de l'institut de linguistique de Louvain, 36(2), 113-133 .

MOÏSE, C., (2016), « Construction de discours sur la sécurité : effets de dramatisation et figures en discours ». In BIGLARI.. A, SALVAN, G. (éds), Figures en discours. Paris, L'Harmattan, 273-292.

PLANTIN, C., (1996), L’Argumentation. Paris: Mémo Seuil.

Rapport d'activité 2017 de l'association Le Refuge [consulté le 10 juin 2018]. Disponible sur le site internet de l'association à l'adresse : www.le-refuge.org.

Rapport annuel 2018 de l'association SOS Homophobie [consulté le 10 juin 2018]. Disponible sur le site internet de l'association à l'adresse, en ligne : https://www.soshomophobie.org/rapport-annuel-2018.

RASTIER, F., (2010), « Témoignages inadmissibles », Littérature, 159,108-129.

RINN, M., (2008), «Introduction ». In RINN, M. (éd.), Emotions et discours. Rennes : Presses Universitaires de Rennes, 13-21

RINN M., (2015), «Introduction. Le corps du témoin comme lieu de parole ». In RINN, M. (éd.), Témoignages sous influence. La vérité du sensible. Presses Universitaires de Laval, 1-8.

ROSIER, L., (2004), « La circulation des discours à la lumière de l'effacement énonciatif: l'exemple du discours puriste sur la langue », Langages, 156, 65-78.

ROSIER, L., (2008), Petit traité de l'insulte. Loverval : Editions Labor.

TIN L.-G. (éd.), (2003), Dictionnaire de l'homophobie. Paris: Presses Universitaires de France.

TORTERAT, F., (2017), « Le témoignage, contre la démémoration \& l'oubli », Acta fabula, 18 (5), Notes de lecture, Mai 2017, en ligne : URL : http://www.fabula.org/acta/document10354.php, page consultée le 2 septembre 2018. 
VAN RAEMDONCK, D., (2011), « Genre, stéréotypes et sexualité ou quand le masculin l'hétéromal - l'emporte ». In DUCHÊNE A. \& MOÏSE C. (éds), Langage, Genre et Sexualité. Paris : Éditions Nota, 173-200.

WEBER, A., (2008), Manuel sur le discours de haine. Leiden, Boston: Martinus Nijoff Publishers. 